\title{
The challenge of insomnia for patients on haemodialysis
}

Daniel Cukor ${ }^{\boxplus}$, Mark Unruh ${ }^{2,3}$, Susan M. McCurry ${ }^{4}$ and Rajnish Mehrotra $\mathbb{( D}^{5}$

Insomnia is common among patients on maintenance haemodialysis and may be exacerbated by the challenges of the COVID pandemic. However, data on the efficacy of insomnia interventions in this population are limited. Efforts are needed to address this important problem and increase access to insomnia interventions for patients on haemodialysis.

More than three million people with kidney failure are currently being treated with maintenance dialysis worldwide. Although this therapy is lifesaving, it is associated with a high treatment and symptom burden. Threats to quality of life include high levels of fatigue, cramps, depression, anxiety and sleep difficulty. Estimates suggest that $40-85 \%$ of patients on dialysis have sleep disturbances ${ }^{1}$. A study that included 1,643 patients from 335 US dialysis centres reported that $50 \%$ had trouble falling asleep, 59\% woke during the night, and $49 \%$ woke early in the morning; $53 \%$ reported one or more of these symptoms all or most of the time ${ }^{2}$.

Insomnia is the most commonly occurring sleep disorder, both in the general population and in patients with kidney failure, and is characterized by persistent difficulty in falling asleep or staying asleep and poor subjective sleep quality. Clinical diagnosis is based on two primary components: sleep difficulties that occur despite adequate opportunities for normal sleep and daytime impairment that directly results from poor sleep quality or duration. Insomnia is considered chronic if it occurs at least three times per week for at least 3 months.

'The Rogosin Institute, New York, NY, USA.

${ }^{2}$ Division of Nephrology, Department of Internal Medicine, University of New Mexico, Albuquerque, NM, USA.

${ }^{3}$ Nephrology Section, New Mexico Veterans Hospital, Albuquerque, NM, USA.

${ }^{4}$ Child, Family, and Population Health Nursing, University of Washington, Seattle, WA, USA. ${ }^{5}$ Kidney Research Institute, Division of Nephrology, University of Washington, Seattle, WA, USA.

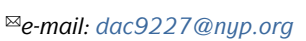
https://doi.org/10.1038/ s41581-021-00396-5

\section{Kidney failure and insomnia}

Insomnia may be of particular concern in patients with kidney failure because of its association with fatigue and poor overall quality of life as well as its potential to lead to various other complications including depression, impaired immune response and higher risk of cardiovascular complications ${ }^{3}$. Patients treated with haemodialysis have ranked sleep difficulty as one of the major threats to their quality of life $\mathrm{f}^{4}$.

Both biological and behavioral factors might contribute to the high prevalence of insomnia in patients with kidney failure. One of the cardinal manifestations of uraemia is day-night reversal. Kidney failure is characterized by substantial changes in circadian rhythm, sleep architecture and endogenous melatonin release. Evidence suggests a direct association between severity of sleep problems and biochemical measures of kidney failure ${ }^{5}$.
In addition, patients receiving in-centre haemodialysis might have a substantially shorter total sleep time and sleep efficiency with greater sleep fragmentation than patients with kidney failure who are treated with other modalities. One third to one quarter of patients who receive in-centre hemodialysis are scheduled to begin treatment in the early morning, often necessitating a rise time of 3-4 a.m., and a similar proportion may end treatment late in the evening, leading to chronic irregularities in sleep schedule. Data suggest that half of all patients nap during thrice weekly haemodialysis sessions, further disrupting their sleep routines ${ }^{6}$. Insomnia in patients on maintenance dialysis can also be exacerbated by other commonly co-occurring sleep disorders such as sleep apnoea and restless leg syndrome.

\section{Coronasomnia}

The COVID pandemic has greatly impacted the dialysis community ${ }^{7}$. In addition to concerns regarding the high rates of morbidity and mortality among patients on maintenance dialysis, stress related to the need to travel to dialysis centres, substantial social isolation, financial instability, housing insecurity, and changes to daily routines have the potential to exacerbate sleep difficulties. The ways that people eat, exercise, relax, relate to each other and sleep have all been impacted by the need for social distancing. Furthermore, patients may find that the people in their social networks who are their usual sources of support are also experiencing stress reactions and sleep problems owing to the challenges of the COVID pandemic. This issue has been dubbed 'coronasomnia.' The extent of the resulting increase in rates of insomnia among patients with kidney failure and its impact on their overall health status and quality of life is not yet known, but insomnia is a risk factor for developing depression and depression often negatively impacts sleep cycle ${ }^{6}$. During these challenging times, the medical community may need to be even more vigilant in addressing insomnia. 
Most

patients with

kidney failure

who experience

sleep difficulty do

not receive any

treatment

\section{Treatment of insomnia}

Given the high frequency and severity of symptoms as well as the unique disease-specific and treatment-specific contributors, a compelling need exists to understand the efficacy of various approaches for treating insomnia in patients on maintenance dialysis.

The American College of Physicians recommends cognitive behavioral therapy for insomnia (CBT-I) as a first line treatment and pharmacological intervention as an adjunct or secondary treatment for insomnia ${ }^{8}$. The core components of CBT-I are stimulus control, sleep restriction and consolidation, cognitive restructuring and sleep hygiene. Stimulus control refers to techniques that are designed to re-establish the association between bed and sleep. These recommendations may include using the bedroom only for sleep or sex, sleeping only in the bedroom and leaving the bed when not able to sleep. The goal of sleep restriction and consolidation is to engender efficient sleep where total sleep time matches the time in bed. Typically, fixed sleep and wake times are established and patients are encouraged to maintain a fixed sleepwake schedule to help get themselves into a healthy sleep rhythm. Cognitive restructuring challenges dysfunctional patient beliefs and attitudes about sleep that can contribute to cognitive hyperarousal at night. Sleep hygiene describes a broad set of 'good sleep habits' that include exercising regularly, avoiding excessive liquids, caffeine, nicotine and alcohol in the evening, ensuring that the bedroom is comfortable and noise-free, and adjusting the timing of meals and snacks relative to bedtime.

Despite clinical practice guidelines that recommend use of CBT-I, pharmacotherapy is often used as a firstline treatment for insomnia. However, commonly used sleep medications have been insufficiently studied in patients on dialysis and concerns exist regarding their efficacy and safety in these patients. Benzodiazepines and non-benzodiazepine receptor agonists are commonly prescribed for patients on haemodialysis even though their use has been reported to be associated with a significantly higher risk of death in this population'.

Most patients with kidney failure who experience sleep difficulty do not receive any treatment. Despite the efficacy of CBT-I, substantial barriers limit its use in clinical practice, both in general and in dialysis centres. Practitioners trained in CBT-I are scarce and are not readily accessed by patients undergoing maintenance dialysis who already have an extremely high treatment burden. Furthermore, CBT-I recommendations might need to be tailored for the unique circumstances of these individuals. Although sleep medications are commonly available, many practitioners are reluctant to prescribe them for chronic insomnia owing to the limited safety and efficacy data for patients with kidney failure.

We are currently investigating the comparative effectiveness of CBT-I administered remotely through telehealth or trazodone versus medication placebo in a randomized controlled trial in patients on maintenance dialysis ${ }^{10}$. Population surveys suggest that trazodone is one of the most commonly prescribed drugs for insomnia in the USA, including among patients with kidney failure but its safety and efficacy have never been studied in patients on long-term dialysis. This trial aims to generate much-needed data regarding effective treatments for insomnia in patients with kidney failure.

\section{Conclusions}

Insomnia is a major concern for patients with kidney failure and may be due to a variety of aetiological causes that span medical, psychological and behavioral domains. As insomnia is associated with negative health outcomes and poor quality of life in patients with kidney disease, the nephrology community needs to be more aware of this problem and work toward making interventions more readily accessible.

1. Roumelioti, M. E. Argyropoulos, C. P. \& Unruh, M. L. in Psychosocial Aspects of Chronic Kidney Disease (eds Cukor, D., Cohen, S. \& Kimmel, P.) 183-212 (Academic Press, 2020)

2. Anand, S. et al. Physical activity and self-reported symptoms of insomnia, restless legs syndrome, and depression: the comprehensive dialysis study. Hemo. Int. 17, 50-58 (2013).

3. Morin, C. M. et al. Insomnia disorder. Nat. Rev. Dis. Primers 1, $1-18$ (2015).

4. Flythe, J. E. et al. Symptom prioritization among adults receiving in-center hemodialysis: a mixed methods study. CJASN 13, 735-745 (2018).

5. Perl, J., Unruh, M. L. \& Chan, C. T. Sleep disorders in end-stage renal disease: 'markers of inadequate dialysis?'. Kid. Int. 70, 1687-1693 (2006).

6. Maung, S. et al. Sleep disturbance and depressive affect in patients treated with haemodialysis. J. Ren. Care 43, 60-66 (2017).

7. Lee, J. et al. Psychosocial impact of COVID-19 pandemic on patients with end-stage kidney disease on hemodialysis. Kidney360 1, 1390-1397 (2020).

8. Brasure, M. et al. Psychological and behavioral interventions for managing insomnia disorder: an evidence report for a clinical practice guideline by the American College of Physicians. Ann. Intern. Med. 165, 113-124 (2016).

9. Winkelmayer, W. C., Mehta, J. \& Wang, P. S. Benzodiazepine use and mortality of incident dialysis patients in the United States. Kid. Int. 72, 1388-1393 (2007).

10. Unruh, M. et al. Sleep-HD trial: short and long-term effectiveness of existing insomnia therapies for patients undergoing hemodialysis. BMC Nephrol. 21, 1-12 (2020).

\section{Competing interests}

The authors declare no competing interests. 